\title{
Comparación de fórmulas chilenas e internacionales para valorar el arbolado urbano
}

\author{
Comparison of Chilean and foreign formulae for urban tree appraisal
}

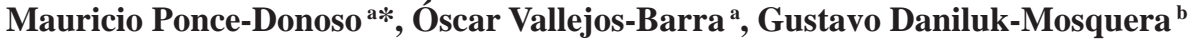 \\ *Autor Correspondencia: ${ }^{\text {a }}$ Universidad de Talca, Facultad de Ciencias Forestales, \\ casilla 747, Talca, Chile, tel.: 56 - 71 - 200432, mponce@utalca.cl \\ ${ }^{\text {b } U n i v e r s i d a d ~ d e ~ L a ~ R e p u ́ b l i c a, ~ U r u g u a y . ~}$
}

\begin{abstract}
SUMMARY
The appraisal of urban trees is a practice adopted in diverse cities of world. This survey compared international formulae: Council of Tree Landscape Appraiser (CTLA), Burnley, Helliwell and Standard Tree Evaluation Method (STEM) and three Chilean methods applied in municipalities of Concepción, La Pintana, Maipú (COPIMA), Ñuñoa and Peñalolén, in 14 different trees located in Talca city (Chile). The objective was to identify the differences and similarities of the monetary result in the application of these formulae, which was realized by a professional. These were analyzed using a non parametric variance test of Kruskal - Wallis and the multiple comparisons Duncan test. It was possible to determine that the Chilean formulae did not present statistically significant differences with the international formulae of Burnley and CTLA; whereas Peñalolén and COPIMA formulae did not present any difference when contrasted with Helliwell. In addition, the STEM formula presented differences with all the Chilean analyzed formulae. In the valuation by tree, statistically significant differences were obtained, which showed the independence of the used formula. The exception was when being applied to emblematic species or to species that stand-out in some amenity. Likewise, it was observed that the basic value continues having a high impact in the appraisal final result and the use of the statistical test applied allows extending this type of analyses.
\end{abstract}

Key words: tree appraisal, non parametric analysis, urban forest, urban forestry.

\section{RESUMEN}

La valoración del arbolado urbano es una práctica adoptada en diversas ciudades del mundo. Este estudio comparó las fórmulas internacionales de Council of Tree and Landscape Appraiser (CTLA), Burnley, Helliwell y Standard Tree Evaluation Method (STEM), y tres chilenas que se aplican en los municipios de Concepción, La Pintana y Maipú (COPIMA), Ñuñoa y Peñalolén, en 14 árboles diferentes, ubicados en la ciudad de Talca (Chile). El objetivo fue identificar las diferencias y similitudes del resultado monetario de estas fórmulas, realizada por un único profesional. Fueron analizados utilizando la prueba de varianza no paramétrica de Kruskal-Wallis y la prueba de comparación múltiple de Duncan. Se concluyó que las fórmulas chilenas no presentaron diferencias estadísticamente significativas con las fórmulas internacionales de Burnley y CTLA; mientras que la de Peñalolén y COPIMA no la tuvo con Helliwell. Por su parte, la fórmula STEM es la que presentó diferencias con todas las fórmulas chilenas analizadas. En la valoración por árbol se obtuvieron diferencias estadísticamente significativas, independientemente de la fórmula utilizada, siendo la excepción cuando corresponde a especies emblemáticas o destacan en alguna amenidad. Asimismo, se observó que el valor base sigue teniendo un alto impacto en el resultado final de la valoración y el uso de la prueba estadística no paramétrica amplía el análisis de las fórmulas.

Palabras clave: valoración del árbol, análisis no paramétrico, arbolado urbano, silvicultura urbana.

\section{INTRODUCCIÓN}

El concepto de arbolado urbano se inicia en Norteamérica en los '60 y en Europa en los'80 (Piolet et al. 2002, Konijnendijk et al. 2005). Por lo general, se identifica como arbolado urbano a diferentes sitios, entre ellos cinturones verdes, líneas de árboles en aceras de áreas residenciales y comerciales, parques, árboles en bandejones, en áreas industriales como también aquellos lugares ubicados en las áreas bajas de las cuencas de las ciudades y otros espacios urbanos donde es posible encontrarlos (Wenger 1984).
El arbolado urbano contribuye al desarrollo sostenible, al considerar aspectos ambientales, sociales y económicos (Tyrväinen 2001, Cullen 2002, Nowak et al. 2002, Tyrväinen et al. 2003, Konijnendijk et al. 2004, 2005, Ponce-Donoso et al. 2009). Diversos autores señalan la dificultad para determinar el aporte que estos bienes son capaces de proveer a través de los procesos de valoración clásicos; sin embargo, es posible identificar variados estudios y propuestas relacionadas con la determinación de un valor monetario relacionado al arbolado urbano (Watson 2002, Tyrväinen et al. 2003), ya sea usando formulas u otras metodologías como valoración contingente, precios hedónicos y costo de viaje. 
La valoración del arbolado urbano no se caracteriza por ser precisa, ya que depende del objetivo de la valoración y del nivel de experticia del evaluador. Su uso o aplicación se lleva a cabo producto de reclamos por daño, litigación en ámbitos judiciales, determinación del patrimonio, entre otros; por lo cual definir su objetivo es relevante. En la mayoría de los "métodos comúnmente utilizados", el valor se define monetariamente con base en la percepción que el "experto" tiene del árbol, que para estos propósitos, la valoración pasa por establecer un criterio mensurable y objetivo, considerando aspectos o variables tales como si se encuentra solo o en grupo, el deterioro físico, grado de impronta, especie y variedad, tamaño, edad, estado y ubicación, entre otras variables que pueden llegar a intervenir (Caballer 1999).

Para la valoración del arbolado es necesario un balance entre procedimientos econométricos, significancia histórica, componentes medioambientales y las características propias del árbol. Chueca (2001) menciona que el objetivo de las metodologías de valoración se reduce a la subjetividad de las variables, que no es el método en sí mismo, sino que depende del sujeto a evaluar, en este caso el árbol; mientras que Cullen (2007) argumenta lo contrario con el uso de CTLA (Council of Tree and Landscape Appraiser), y consigna que son las variables las que pueden variar a través del tiempo, en cuanto a su importancia o peso en la fórmula.

La metodología para valorar el arbolado urbano ampliamente usada y aceptada a nivel mundial es la aplicación de fórmulas (Watson 2001). Se identifican una o más fórmulas en Argentina, Australia, Chile, Estados Unidos, España, Finlandia, Francia, Gran Bretaña, Nueva Zelanda, entre otros países, de distinta composición y objetivos de aplicación. Asimismo, comparar metodologías de valoración del arbolado urbano continúa siendo un trabajo necesario para su desarrollo y desempeño.

Diversos estudios han comparado fórmulas (Tyrväinen 2001, Watson 2002, Contato-Carol et al. 2008, PonceDonoso et al. 2009). Por ejemplo, Randrup (2005) quien desarrolló una fórmula para Dinamarca, señala que los diferentes modelos varían en forma y por ende en la valoración final del árbol. McPherson (2007) estudió la valoración del arbolado basándose en la relación costo-beneficio; mientras que Leal et al. (2008) relacionaron la inversión en el arbolado urbano con la teoría de la renta de la tierra.

En general, se identifican dos maneras de valorar el arbolado urbano. Una es a través de métodos paramétricos o multiplicativos, es decir, fórmulas que utilizan preferentemente variables de estado y de amenidades de los árboles, las que son vinculadas a ponderaciones o pesos que pueden cambiar en el tiempo; y otras de tipo económicas o llamadas métodos de capitalización. Para Contato-Carol et al. (2008) el método paramétrico es superior al de capitalización, que es especialmente usado en el sector público por su simplicidad, rapidez y eficiencia de aplicación.

Las principales fórmulas estudiadas son: el método del Council of Tree and Landscape Appraiser, desarrollado en Estados Unidos (CTLA 2000); el método Burnley, desarrollado por el Colegio Victoriano de Agricultura y Horticultura de Australia (Moore 1991); el método de valoración de las amenidades de los árboles y bosques de Gran Bretaña, llamado de Helliwell (Watson 2002); el método Standard Tree Evaluation Method (STEM) desarrollado en Nueva Zelanda en 1996; y la Norma Granada, desarrollada por la Asociación Española de Parques y Jardines Públicos (AEPJP 2007). Asimismo, existen métodos en Suiza, Finlandia y Francia (Contato-Carol et al. 2008).

Estudios que comparan fórmulas internacionales fueron realizados por Watson (2002) y Contato-Carol et al. (2008). La principal contribución de Watson (2001) es señalar que la subjetividad de los tasadores generaban diferencias de importancia en las evaluaciones, de igual modo determinó que CTLA y Burnley obtienen los valores más bajos, Helliwell el más alto y STEM tuvo la variación más baja entre tasadores, señalando que las variables y su efecto multiplicativo aumentarían estas diferencias. Contato-Carol et al. (2008) aplicaron cinco fórmulas en árboles de la ciudad de Santiago del Estero, Argentina, obteniendo el método suizo y finlandés las valoraciones más altas, CTLA entrega un valor moderado como el francés, resultando ser similares; mientras que el de capitalización fue considerado el más objetivo por los autores, a pesar de que los valores entre ejemplares son los que más variaron.

En Chile la valoración del arbolado urbano, preferentemente ubicados en espacios de uso público, se encuentra ligada a la determinación del daño de los árboles y a la existencia de normativas municipales que incluyan la valoración (Ponce-Donoso et al. 2009). Su aplicación y estudio son aún incipientes. Generalmente se realiza con participación de personal municipal, de acuerdo a la ley Orgánica Constitucional de Municipalidades (Ministerio del Interior 2004) que le corresponde proponer una multa a aplicar por parte del juez de policía local, a partir de la forma de valoración incluida en la normativa respectiva. Cullen (2005) releva el rol que les compete a estos tasadores en los procedimientos que se incoan en los juzgados, responsables de la propuesta de valoración. Ponce-Donoso et al. (2009) señalan que las ordenanzas municipales chilenas no son claras, ya que no se distingue la idea de aplicación de multa y el resarcimiento por daños a los bienes públicos, es decir, al arbolado.

Las fórmulas existentes presentan diferencias y similitudes en su composición y resultados, por ello, el objetivo de este trabajo fue comparar los valores monetarios obtenidos al aplicar tres fórmulas que se usan en cinco comunas de Chile y cuatro fórmulas usadas en otros países, determinando la existencia de diferencias estadísticamente significativas entre fórmulas y ejemplares estudiados. $\mathrm{La}$ aplicación de siete fórmulas en 14 especies de árboles urbanos totaliza 98 valoraciones. El trabajo presentado es la continuación del desarrollado por los autores que identi- 
ficaron fórmulas aplicadas en Chile y busca contribuir a generar información para la construcción de una fórmula destinada al país.

\section{MÉTODOS}

El estudio consideró el arbolado urbano público en la ciudad de Talca, región del Maule, Chile. La ciudad se encuentra a una altitud de $102 \mathrm{~m} \mathrm{snm}$, latitud 35 25' 59' Sur y longitud $71^{\circ} 40^{\prime} 00^{\prime \prime}$ Oeste. Cubre una superficie de 51 $\mathrm{km}^{2}$ y posee una población urbana cercana a los 170 mil habitantes.

Se seleccionaron 14 árboles de los considerados por Ponce-Donoso et al. (2009), que tuvo como base: a) especie nativa o exótica, considerando si es emblemática, término que se entiende como una especie sobre los 50 años, endémica, de connotación histórica o botánica, u otra característica que sola o en combinación permitan identificar esa condición; b) especies con mayor frecuencia en la ciudad de Talca; y c) edad de la especie; teniendo la certeza que las variables pudieran medirse y de contar con información que se pudiera recabar desde el Departamento de Sistemas de Información Geográfica de la Ilustre Municipalidad de Talca. El número de especímenes usado fue considerado adecuado, si se tiene en cuenta los estudios de Watson (2001) y Contato-Carol (2008) que no superaron los ocho árboles. De esta manera, el número total de evaluaciones permite aplicar el análisis estadístico propuesto. El anexo 1A muestra las características de los árboles analizados.

El trabajo de campo se desarrolló en dos etapas. La primera incluyó la medición de las variables dendrométricas como DAP, altura, perímetro del dosel, y de otras como valores estéticos, condición sanitaria, ubicación y resto de las variables usadas, que fue realizado entre los meses de septiembre y octubre de 2007. El segundo correspondió a la determinación de antecedentes monetarios, tales como referencias de precios de venta en viveros locales, tanto al por mayor como menor, costos de mantenimiento anual y de extracción de árboles, información que fue proporcionada por el Departamento de Aseo y Ornato de la Ilustre Municipalidad de Talca. Los costos de mantenimiento fueron calculados a base de los costos anuales de poda y otros manejos para un total de 2.000 árboles al año y oscilan entre US\$24,3 y US\$ 17,8 por árbol, respectivamente, los que fueron actualizados usando la unidad tributaria mensual (UTM) ${ }^{1}$ (Municipalidad de Talca 2006). Sin embargo, se debe considerar que estos costos varían de modo significativo entre municipios, dependiendo de las necesidades de mantenimiento, de las estructuras de contrato con los proveedores de servicios y la capacidad de gestión del municipio. Para el precio en vivero de las especies, se usó el de mercado y las alturas promedio de venta al por menor, teniendo como referencia viveros ubicados en las regiones ${ }^{1}$ UTM corresponde a una unidad monetaria reajustada mensualmente por el índice
de precios al consumidor (IPC), que en este estudio correspondió a US\$ 68,24. del Maule, de O’Higgins y Metropolitana, consultándose un total de 12 viveros (anexo 1B).

Se aplicaron las fórmulas nacionales ${ }^{2}$ utilizadas por los municipios de Concepción, La Pintana, Maipú, Ñuñoa y Peñalolén (fórmulas a, b, c), y las fórmulas internaciona$\operatorname{les}^{3}$ (d, e, f, g), descritas a continuación.

a) Municipalidades de Concepción, La Pintana y Maipú (denominada en este estudio COPIMA).

Valor $(\mathrm{US} \$)=(\mathrm{A} * \mathrm{~B} * \mathrm{C} * \mathrm{D}) / 10$

Donde:

$\mathrm{A}=$ corresponde al precio de la especie en el mercado minorista. Las especies deben tener entre 12 a $14 \mathrm{~cm}$ de perímetro a la altura del cuello. La altura considerada para las especies de hoja persistente es de 3,5 a 4,0 m y para confieras y palmeras de 2,0 a $2,5 \mathrm{~m}$.

$\mathrm{B}=$ valor estético y de sanidad del árbol. El rango va de 1 a 10 y considera, dependiendo de la belleza del árbol, la relación con otros árboles, protección, sanidad, vigor y valor dendrológico. El coeficiente será el siguiente: 10 para sano, vigoroso, solitario y destacable; 9 para sano, vigoroso, en grupo de 2 a 5 destacables; 8 para sano vigoroso, en grupo, en cortina o alineación (hilera); 7 para sano, vegetación mediana, solitario; 6 para sano, vegetación mediana, en grupo de 2 a 5; 5 para sano, vegetación mediana, en grupo, cortina o alineación (hilera); 4 para poco vigoroso, envejecido, solitario en su alineación (hilera); 3 para sin vigor, en grupo, mal formado; 2 para sin vigor, enfermo, sólo en alineación (hilera); y 1 para sin valor.

$\mathrm{C}=$ índice de situación. Aspecto relacionado con la evaluación ambiental y la urbanización. Puede alcanzar valores de 6 para zonas rurales o agrícolas; 8 en barrios y 10 en el centro urbano.

$\mathrm{D}$ = índice de dimensión. Perímetro del árbol medido a 1,3 $\mathrm{m}$ sobre el nivel del suelo. Los valores son:

1 hasta $30 \mathrm{~cm}$; 3 desde 30,1 a los $60 \mathrm{~cm}$; 6 desde 60,1 a los $100 \mathrm{~cm}$; 9 desde 100,1 a los $140 \mathrm{~cm} ; 12$ desde 140,1 a los $190 \mathrm{~cm} ; 15$ desde 190,1 a los 240 cm; 18 desde 240,1 a los $300 \mathrm{~cm}$; y 20 mayor de $300 \mathrm{~cm}$.

\section{b) Municipalidad de Ñuñoa}

Valor $(\mathrm{UTM})=\mathrm{VA} * \mathrm{DO} *(1-\mathrm{DP})$

Donde:

$\mathrm{VA}=$ precio de la especie en el mercado expresada en UTM, de acuerdo con el rango de edad y el grupo de especie a la que pertenece, que se clasifican en tres grupos (anexo 1C y anexo 2).

Las especies valoradas del grupo 1 son: Acer negundo L.,

\footnotetext{
${ }^{2}$ Para mayores antecedentes remitirse a: Ponce-Donoso et al. (2009).

${ }^{3}$ Para mayores antecedentes remitirse a: Watson (2001). La descripción detallada de cada una de las fórmulas fue obviada debido a la amplia difusión y conocimiento de ellas.
} 
Ailanthus altisima Mill., Grevillea robusta A. Cunn., Melia azedarach L y Catalpa bignonioides Walter; del grupo 2: Jacaranda mimosifolia D. Don y Liquidambar styraciflua L.; y del grupo 3: Cryptocarya alba (Mol.) Looser, Quillaja saponaria Mol., Schinus areira L., Ginkgo biloba L., Jubaea chilensis (Mol.) Baillon, Quercus spp. y Tilia spp. $\mathrm{DO}=$ daño causado. Valor que va entre 0 y 1 , y es interpretado como la pérdida de los atributos estéticos, funcionales y fisiológicos.

DP = daño presente. Valor entre 0 y 1 , representa el deterioro presente del estado estructural y fisiológico del árbol, causado por agentes físicos y biológicos.

c) Municipalidad de Peñalolén

Valor $(\mathrm{UTM})=(0,20 \mathrm{UTM} * \mathrm{e})+(\mathrm{A} * \mathrm{~B} * \mathrm{VA})$

Donde:

0,20 UTM = costos de mantenimiento anual del árbol.

$\mathrm{e}=$ edad de la especie en años

A = factor de ubicación. Relaciona la ubicación del árbol en las distintas áreas de la ciudad. Para plazas, parques o áreas verdes $200 \%$; calles o avenidas $100 \%$ del valor del árbol.

$\mathrm{B}=$ estado del árbol. Definido como el porcentaje de daño del árbol, con daño entre el 20 al 80\%, el factor de ponderación es 0,8 .

VA = valor del árbol. Acorde con la especie y su edad, la que es multiplicada por un factor (anexo 1C y anexo 3).

Las especies valoradas usadas en este estudio son para el grupo 1: Acer negundo L., Ailanthus altisima Mill., Grevillea robusta A. Cunn. ex R.Br, Melia azedarach L., Tilia spp. y Catalpa bignonioides Walter; en el grupo 2: Ginkgo biloba L., Jacaranda mimosifolia D. Don, Liquidambar styraciflua L., Quillaja saponaria Mol., Cryptocarya alba (Mol.) Looser, Quercus robur L. y Schinus areira L.; mientras que la del grupo 3 es: Jubaea chilensis (Mol.) Baillon.

d) Council of Tree and Landscape Appraisers de Estados Unidos (CTLA). El método considera el área de la sección transversal del tronco a 1,4 m sobre el nivel del suelo, multiplicado por un valor basado en el costo de la disponibilidad de la especie en viveros regionales. El valor es multiplicado por factores tales como condición, ubicación y calidad, usando una ponderación entre 0,0 a 1,0 para cada factor. La fórmula es la siguiente:

Valor $\left(\right.$ US\$) $=\left(\right.$ área del tronco $\left(\mathrm{cm}^{2}\right)^{*}$ precio básico $\left./ \mathrm{cm}^{2}\right)$

* especie * condición * localización

e) Burnley (Australia). El método utiliza el tamaño del árbol (medido como el volumen de un cono invertido) y un valor monetario, denominado valor base. El volumen del árbol es multiplicado por el costo por metro cúbico en viveros minoristas, de la misma especie preferentemente. El valor es modificado por factores que podrían reducir el valor base: expectativa de vida $(0,5$ a 1,0$)$; forma y vigor $(0,0$ a 1,0); y ubicación $(0,4$ a 1,0). La fórmula es la siguiente:

Valor (US\$) = volumen del árbol * valor base * expectativa de vida * forma y vigor * localización

f) Helliwell (Amenity Valuation of Trees and Woodlands, de Gran Bretaña). Este método valoriza las amenidades visuales y usa puntos de 1,0 a 4,0 para siete factores, a saber: tamaño del árbol, expectativa del vida, importancia en el espacio, presencia de otros árboles, relación con el entorno, forma y factores especiales (en algunos casos puede ser menor que 1,0), que son en conjunto multiplicados por un valor monetario determinado ( $£ 14$, catorce libras esterlinas), equivalente a US\$21,2. La fórmula se expresa como:

Valor $($ US\$) $=$ tamaño del árbol * expectativa de vida * importancia en el espacio * presencia de otros árboles * relación de otros factores $*$ forma especial $*$ monto en dinero

g) Standard Tree Evaluation Method, de Nueva Zelanda (STEM). Esta fórmula utiliza un sistema de puntos a base de veinte atributos ( 3 a 27 puntos para cada uno) en tres campos: condición, amenidad y características especiales de notabilidad. Los atributos son: condición (forma, frecuencia, vigor y vitalidad, función, y edad); amenidades (estatura, visibilidad, presencia de otros árboles, papel, y clima); notabilidad, solo para los árboles que tengan más de 50 años de edad (estatura: aspecto, y forma; historia: edad, asociación, conmemoración, remanente, y relicto; y científico: fuente, rareza, y riesgo). El total de puntos es multiplicado por el precio mayorista en vivero de un árbol de cinco años de edad preferiblemente, más el costo mayorista de plantación y mantenimiento del árbol a la misma edad que éste se hubiera perdido, de esta manera, esto se multiplica por un factor de conversión desde mayorista a minorista, para lo cual se recomienda un valor 2. La fórmula es la siguiente:

Valor (US\$) $=$ [total de puntos (540 posibles) $*$ costo mayorista + costo plantación + costo mantención] * factor de conversión minorista (sugerido 2)

Las variables medidas para cada una de las fórmulas se muestran en el anexo 1D. Para el análisis de los datos se utilizó el promedio y la mediana de la valoración, tanto para árbol como fórmula; este último estadígrafo ayuda a reducir el impacto de los valores extremos.

Se plantearon las siguientes hipótesis para las fórmulas de valoración y los ejemplares:

- $\mathrm{H}_{0}: \omega_{\mathrm{i}}=\omega_{\mathrm{j}} / \mathrm{i} \neq \mathrm{j}$; (es decir, no existen diferencias estadísticamente significativas entre las medianas de las fórmulas y de ejemplares considerados). 
- $\mathrm{H}_{1}: \omega_{\mathrm{i}} \neq \omega_{\mathrm{j}} / \mathrm{i} \neq \mathrm{j}$; (es decir, existen diferencias estadísticamente significativas entre las medianas de las fórmulas y de ejemplares considerados).

Se utilizó análisis de varianza para determinar si existen diferencias estadísticamente significativas entre las fórmulas de valoración del arbolado urbano y entre los ejemplares analizados. Dado que los supuestos de este análisis no se cumplieron (homocedasticidad y normalidad), ya que se obtuvo un elevado coeficiente de variación $(38,9 \%)$ y que el sesgo y curtosis estandarizado (40,9 y 150,8 respectivamente) superaron el valor límite establecido de 2,0, no se pudo trabajar aplicando estadística paramétrica, inclusive, aplicando las transformaciones sugeridas por Kirk (1995). En estos casos, se debe recurrir al análisis de varianza no paramétrico de Kruskal-Wallis (Conover 1999) que es menos sensible a la presencia de valores atípicos. Este análisis comprueba la existencia de diferencias estadísticamente significativas entre las medianas del ranking. Los valores originales son transformados a un ranking dependiendo de su posición en el ordenamiento ascendente de los datos, es decir, el ranking 1 le corresponde a la menor valoración (US\$ 1,9) y el ranking 98 a la mayor valoración (US\$ 34.551,5). Los ranking intermedios corresponden a las valoraciones intermedias. De existir diferencias estadísticamente significativas entre las diferentes fuentes de variación, se recurrió a la prueba de comparación múltiple de Duncan descrita por Conover (1999) para identificar fuentes de variación disímiles. Para el análisis de los datos se utilizaron los programas Microsoft Excel v.2003 y Statgraphics Centurion v.XVI, ambos para Windows.

\section{RESULTADOS}

Considerando de manera individual el valor promedio y la mediana de la valoración para cada árbol (cuadro 1), se observó que los resultados son los esperables, teniendo presente sus características particulares. Una de ellas es la notabilidad de la especie, que originó los valores más altos, como fueron los casos de los árboles G. biloba y J. chilensis. Asimismo, los valores medios fueron influenciados por la ubicación, las características sanitarias, junto con las demás variables, a excepción de la notabilidad, en los valores de los árboles A. altisima, C. alba, J. mimosifolia y Q. saponaria. En cambio, en aquellos donde el árbol presentaba estas variables disminuidas, debido principalmente a problemas estructurales y fitosanitarios, tuvieron valores bajos, como fueron los casos de los árboles C. bignoniodes, T. americana y Q. robur, aunque este último fue sobrevalorado por STEM, debido a su localización y para T. americana su valor fue mayor producto de la ausencia de problemas fitosanitarios o estructurales como los referidos en las demás especies.

Cuadro 1. Valor monetario aplicando las fórmulas propuestas (US\$). Appraisal output (US\$).

\begin{tabular}{|c|c|c|c|c|c|c|c|c|c|}
\hline \multirow{2}{*}{$\begin{array}{c}\text { Árbol } \\
n^{\circ}\end{array}$} & \multicolumn{9}{|c|}{ Fórmulas } \\
\hline & CTLA & Helliwell & Burnley & STEM & COPIMA & Ñuñoa & Peñalolén & Promedio & Mediana \\
\hline 1 & 169,6 & 127,2 & 0,8 & $1.376,8$ & 247,2 & 160,3 & 341,2 & 346,2 & 169,58 \\
\hline 2 & 676,2 & $1.017,6$ & 409,1 & $2.302,5$ & 672,0 & 360,8 & 382,2 & 831,5 & 672,00 \\
\hline 3 & 56,8 & 763,2 & 3,2 & $1.306,9$ & 89,6 & 3,5 & 341,2 & 366,3 & 89,59 \\
\hline 4 & $1.766,5$ & $27.475,2$ & 628,9 & $3.952,5$ & 624,0 & 668,9 & $1.433,0$ & $4.061,4$ & $1.433,04$ \\
\hline 5 & $16.930,2$ & $8.140,8$ & $1.298,5$ & $11.547,4$ & $4.320,0$ & $1.228,6$ & 1,433,0 & 4.989,2 & $4.320,00$ \\
\hline 6 & 427,7 & 954,0 & 332,5 & $2.547,2$ & 545,2 & 13,2 & 245,7 & 723,6 & 427,72 \\
\hline 7 & $1.314,6$ & $6.105,6$ & 384,3 & $6.565,3$ & $1.684,8$ & 789,6 & $1.433,0$ & $2.031,0$ & $1.433,04$ \\
\hline 8 & $17.311,8$ & $34.344,0$ & $1.269,7$ & $17.842,0$ & 7.919,9 & 413,3 & 341,2 & $11.348,8$ & 7.919,91 \\
\hline 9 & 272,4 & 254,4 & 9,4 & $3.716,9$ & 665,6 & 56,8 & 218,4 & 742,0 & 254,40 \\
\hline 10 & 464,7 & $2.544,0$ & 53,5 & $1.754,8$ & 460,8 & 47,6 & 341,2 & 809,5 & 460,80 \\
\hline 11 & 4,2 & 15,9 & 0,2 & $1.226,9$ & 39,0 & 3,6 & 68,2 & 193,9 & 15,90 \\
\hline 12 & $1.368,7$ & $1.272,0$ & 80,1 & $2.564,9$ & 616,4 & 10,1 & 341,2 & 893,3 & 616,38 \\
\hline 13 & 127,9 & 106,0 & 40,4 & $1.287,8$ & 119,1 & 371,9 & 341,2 & 342,0 & 127,90 \\
\hline 14 & 5,7 & $2.289,6$ & 14,8 & $1.603,0$ & 43,2 & 80,3 & 68,2 & 586,4 & 68,24 \\
\hline Promedio & $2.921,2$ & $6.100,7$ & 323,2 & $4.256,7$ & $1.289,1$ & 300,6 & 523,5 & & \\
\hline Mediana & 446,2 & $1.144,8$ & 66,8 & $2.424,8$ & 580,8 & 120,3 & 341,2 & & \\
\hline
\end{tabular}

Nota: 1 US\$ = CL \$ 500, aproximadamente. 
STEM obtuvo el monto mayor de la mediana en las 14 evaluaciones aplicadas, que duplicó su valor con la fórmula que le sigue, que correspondió a Helliwell; ambas obtuvieron los valores de las medianas más altas, conformando en sí uno de los grupos homogéneos, cuya mediana promedió un valor de US\$1.784,8, que para el caso de Helliwell logró obtener las dos más altas valoraciones por árbol, C. alba y J. chilensis, correspondientes a especies emblemáticas. Con resultados intermedios de valores en las medianas, se encontraron las fórmulas de COPIMA, CTLA y Peñalolén, que también caracterizaron otro subgrupo, cuyo valor no excedió los US\$ 581,0 como máximo. Cierra el grupo por fórmulas las de Ñuñoa y Burnley que formaron un subgrupo, cuyo valor medio de la mediana fue de US\$ 93,6.

El análisis de varianza de Kruskal-Wallis determinó que las fórmulas de valoración del arbolado y las especies presentaron diferencias estadísticas altamente significativas. El estadígrafo de Kruskal-Wallis tuvo un valor de 35,0 para las fórmulas, mientras que para las especies fue de 44,5, ambos con una probabilidad inferior al $1 \%$ $(P<0,001)$. La identificación de las diferencias se observa en el cuadro 2.

Se agruparon las siete fórmulas en tres grupos homogéneos (figura 1, cuadro 2). Las tres fórmulas chilenas se encuentran ubicadas de manera secuencial con valores comparativamente bajos e intermedios. La fórmula internacional de Burnley aportó el valor más bajo, mientras CTLA y Helliwell compartieron un lugar intermedio y esta última en conjunto con STEM presentaron los valores máximos. Asimismo, al considerar los ejemplares (figura 2, cuadro 2) se observaron cuatro grupos homogéneos. Los menores valores estuvieron referidos a especies con algún tipo de daño, en tanto que las tres especies nativas consideradas, presentaron valores intermedios y altos. Los tres valores

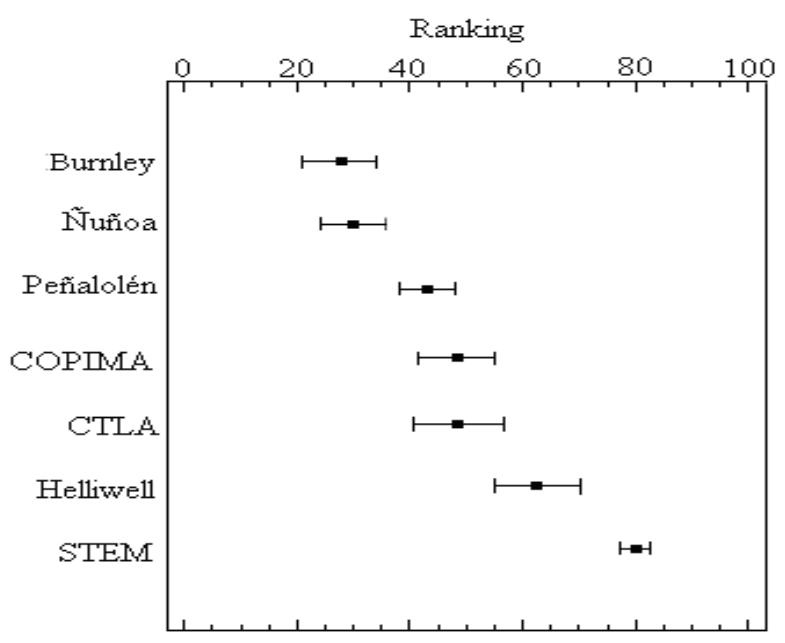

Figura 1. Mediana y su error estándar de cada fórmula de valoración.

Median and stadard error for appraisal formula. máximos lo obtuvieron especies emblemáticas ( $G$. biloba y J. mimosifolia) y de importancia ecológica (J. chilensis).

\section{DISCUSIÓN}

Las variables de estado de los árboles contenidas en las fórmulas generan las principales diferencias de tasación de aquellos que presentan características físicas, sanitarias u otras que los "diferencien” del resto, aunque la variable "valor base”, sigue siendo un aspecto sustantivo en la aplicación de cualquier fórmula. En su determinación es importante incorporar las condiciones del mercado local, pero aún así, resulta ser un elemento significativo en la estructura de la fórmula y la determinación del valor final.

Se determinó que existe correspondencia con los resultados obtenidos por Watson (2002) en relación a la aplicación de las fórmulas internacionales, aunque se presenta una diferencia con el resultado de la fórmula STEM, que se debería a la incorporación de especies notables en este estudio, a diferencia de lo realizado por este. Lo anterior es similar en la aplicación de las fórmulas de Helliwell y CTLA, pero en menor grado con la de Burnley, en cuyo caso se obtuvieron valores promedios comparativamente más bajos. Sin embargo, Watson (2001) y Contato-Carol et al. (2008) obtuvieron valores medios en las tasaciones realizadas con la fórmula CTLA, lo que se debería a las características de menor uso de especies de menor notabilidad.

La posición relativa obtenida en valores promedios indica que se obtienen valores más altos con las fórmulas de Helliwell y STEM, influyendo en el valor las amenidades del árbol y el valor patrimonial, respectivamente. Con las fórmulas CTLA y COPIMA se obtienen valores medios de manera similar a los obtenidos en los otros dos estudios mencionados; mientras que valores menores promedio se

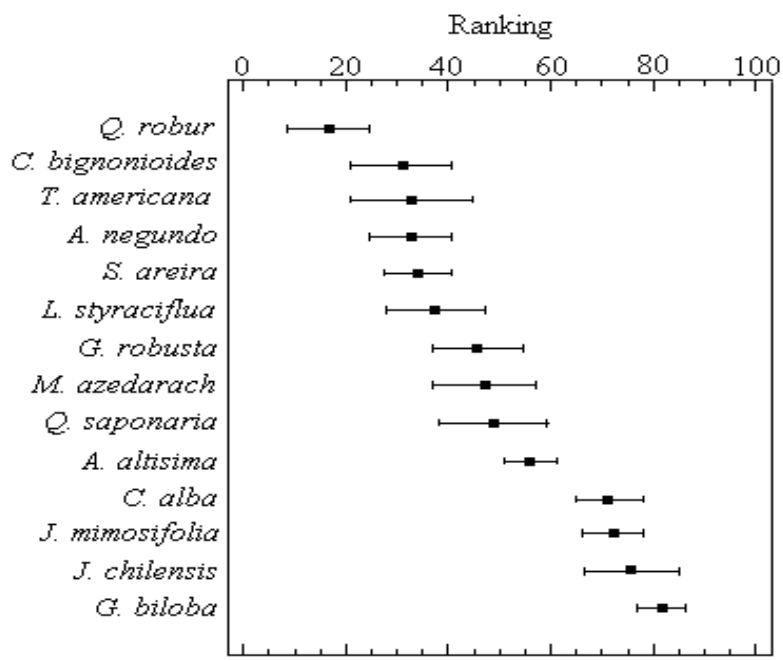

Figura 2. Mediana y su error estándar de cada ejemplar valorado. Median and estandar error for valued species. 
Cuadro 2. Prueba de comparación múltiple de Duncan por ranking para fórmula y especie. Multiple comparison ranking Duncan test for formula and species.

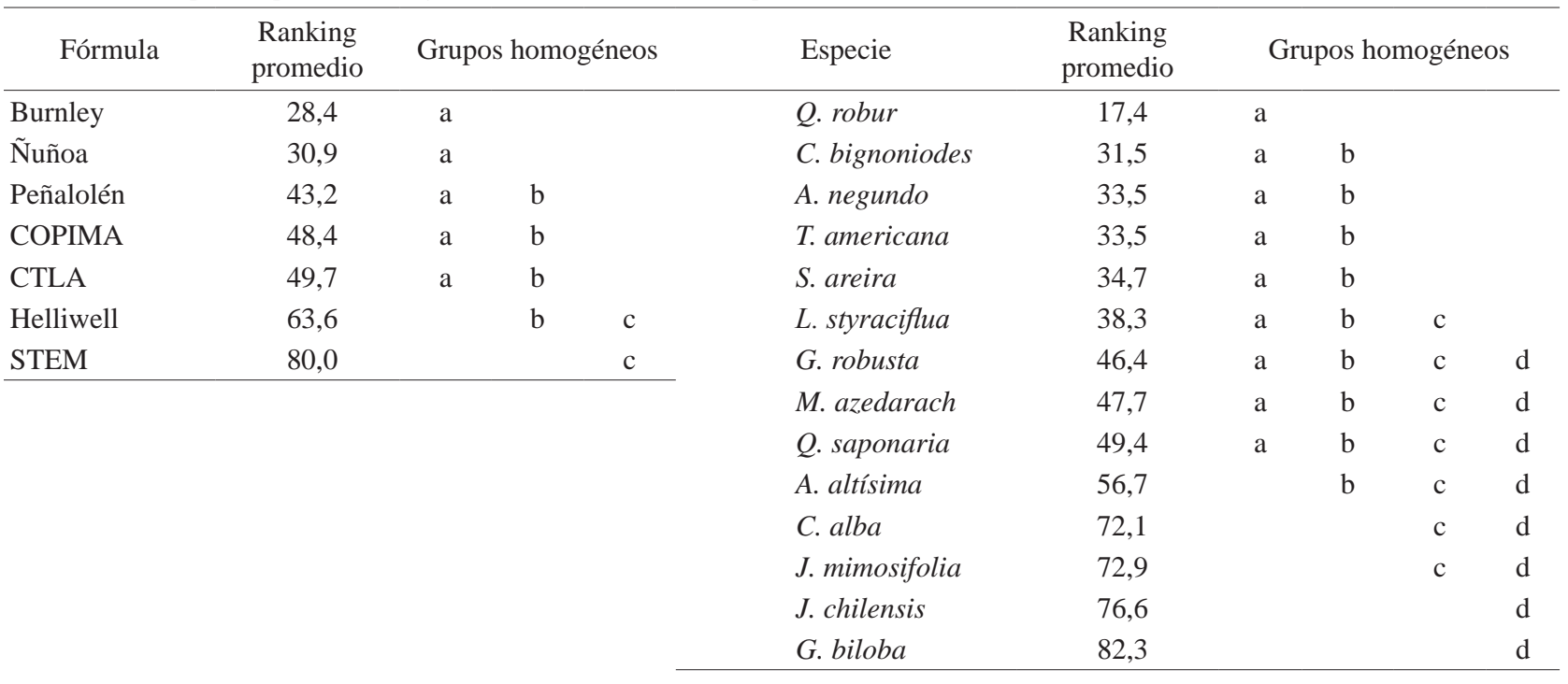

logran al usar las fórmulas de Burnley, Ñuñoa y Peñalolén, siendo el valor obtenido con la fórmula de Burnley menor al alcanzado comparativamente en el estudio de Watson (2002) y Ponce-Donoso et al. (2009).

Las fórmulas internacionales obtienen un valor promedio más alto para todos los árboles (cuadro 2), con la excepción de Burnley, que es superada por las fórmulas chilenas COPIMA y Peñalolén. Significativamente más altos son los valores para Helliwell y STEM, donde las especies emblemáticas alcanzan un valor promedio más alto, J. chilensis con US\$ 11.348,8; G. biloba US\$ 6.414,1 y C. alba US\$ 4.061,4, debido principalmente a las variables atributos de notabilidad que estas fórmulas miden (Watson 2002, Ponce-Donoso et al. 2009). La excepción son las fórmulas de Ñuñoa y Peñalolén, que no incluyen variables relacionadas con la singularidad del árbol, aspecto que destaca Cullen (2002) como importante fuente de variación en la valoración de los árboles. Es posible observar que el valor promedio para la fórmula de Burnley es el más bajo en las fórmulas internacionales, siendo la de Ñuñoa la de menor valor promedio en todos los casos para las fórmulas chilenas estudiadas.

Es posible encontrar (cuadro 1) diferencias para un mismo árbol al aplicar las fórmulas, aspecto que ya destacaba Cullen (2002) en su estudio de los factores componentes de fórmulas y su impacto en el valor del árbol. Este aspecto coincide con los resultados obtenidos por ContatoCarol et al. (2008) y Ponce-Donoso et al. (2009).

Los resultados permiten identificar tres grupos de fórmulas (figura 1). El primero está compuesto por Burnley y Ñuñoa, con valores menores en el ranking y sin diferencias estadísticamente significativas entre ellas. Asimismo, forman otro grupo las fórmulas de Peñalolén, COPIMA y CTLA, que tienen valores intermedios en el ranking; y finalmente
Helliwell, no presenta diferencias con STEM, que conforman el tercer grupo con los mayores valores en el ranking.

Con respecto a los ejemplares estudiados, existen cuatro grupos que no presentan diferencias estadísticamente significativas. Así, G. robusta, M. azedarach y Q. saponaria no presentan diferencias como resultado de la aplicación de las fórmulas; mientras que en el otro extremo están los ejemplares de Q. robur, J. chilensis y G. biloba, en cuyo primer caso se debería a la disminuida condición física y sanitaria del árbol y en las otras dos a sus características emblemáticas.

Los resultados del análisis de comparación múltiple (cuadro 2), son coincidentes con los estudios de Watson (2002) y Contato-Carol et al. (2008), aunque se debe tener en consideración que la naturaleza metodológica planteada por éstos es diferente, aun cuando lo que finalmente se compara son los resultados de la evaluación. Independientemente de la composición de las fórmulas, existen grupos, de fórmulas como de árboles, donde no se presentan diferencias estadísticamente significativas utilizando esta metodología de análisis no paramétrico. Por el contrario, las que más se diferencian son las que presentan carácter emblemático o aquellas de corta edad y con problemas estructurales o sanitarios. Por lo general se evalúan especies con estados físicos y sanitarios que no impactan en su evaluación, sin embargo, los especímenes estudiados Q. robur, J. chilensis y G. biloba; presentan una situación opuesta a lo indicado.

Según Watson (2001), Cullen (2005) y Ponce-Donoso et al. (2009) para lograr entender de mejor la aplicación y actualización de fórmulas de valoración, así como la participación de cada una de las variables en el valor final, incluyendo otras fuentes de variación como es el tasador, se debe continuar con la comparación de los métodos, es- 
pecialmente, si se busca identificar una fórmula que logre evaluar monetariamente de manera adecuada un árbol, en especial cuando se le quiera incorporar otros aspectos relacionados con su funcionalidad desde el punto de vista de su contribución ambiental.

La comparación de fórmulas, ya sea por la vía paramétrica como no paramétrica, debe considerar las diferentes fuentes de variación, entre ellas: fórmula, valor obtenido, evaluador, interacción de éstas, ponderando cada una de ellas para llegar a conclusiones que permitan señalar si éstas presentan o no diferencias en su aplicación, caso contrario sólo se podrá llegar a establecer una posición ordinal respecto del valor final relacionado con los valores promedios obtenidos en la evaluación.

Como limitaciones al estudio deben ser consideradas las siguientes: a) las evaluaciones son aplicadas a base de aspectos apreciativos o subjetivos del evaluador, a partir de las variables que conforman la fórmula, un aspecto habitual de este tipo de valoraciones, que sólo a través de la experticia del evaluador se puede ir disminuyendo; b) la evaluación realizada se restringe a un único evaluador, por lo cual se recomienda la incorporación de un número mayor de evaluadores, que permita ampliar la base de evaluaciones y generar una nueva fuente de variación; y c) dado que las evaluaciones fueron realizadas a 14 árboles, la muestra puede ser limitada, por lo que también es recomendable su ampliación tanto a especies, como al número en cada una de ellas, considerando distintos niveles de desarrollo, condición y ubicación.

\section{CONCLUSIONES}

De acuerdo a los resultados obtenidos, se rechaza la hipótesis nula, dado que se encontraron diferencias estadísticamente significativas en las valoraciones hechas, considerando tanto las fórmulas como los ejemplares evaluados.

Las tres fórmulas chilenas estudiadas, que se aplican en cinco municipios, no presentan diferencias estadísticamente significativas al aplicarse en los 14 ejemplares evaluados. Asimismo, las fórmulas de Peñalolén y COPIMA, ambas chilenas, no presentan diferencias estadísticamente significativas con las internacionales CTLA y Helliwell en los mismo 14 ejemplares valorados.

Las fórmulas de Helliwell y STEM, ambas internacionales, conforman un único grupo que se diferencia del resto de las fórmulas evaluadas, con las mayores diferencias y valores más altos de las evaluaciones realizadas, siendo STEM la que presenta diferencias altamente significativas con todas las fórmulas chilenas.

Los ejemplares evaluados conformaron cuatro grupos homogéneos que presentan diferencias estadísticas significativas de su valoración, ubicándose la mayoría de los ejemplares en más de un grupo, lo que demostró que la aplicación de una u otra fórmula no tiene alto impacto en los resultados obtenidos.
Las fórmulas chilenas se pueden usar de manera indistinta, mientras no se trate de árboles emblemáticos, de corta edad o con daños importantes. Las que presentan menor grado de diferenciación son las fórmulas de Peñalolén y COPIMA.

Finalmente, se requiere ampliar el estudio incorporando mayor número de ejemplares, especies y evaluadores, que posibiliten analizar las diferencias de estas fuentes de variación y sus interacciones.

\section{AGRADECIMIENTOS}

Los autores agradecen el apoyo recibido por la Dirección de Investigación de la Universidad de Talca al proyecto "Valoración del arbolado urbano, desarrollo de una fórmula para Chile Central” y a la Ilustre Municipalidad de Talca por la información proporcionada.

\section{REFERENCIAS}

AEPJP (Asociación Española de Parque y Jardines Públicos, ES). 2007. Norma Granada. Método para valoración de ár-

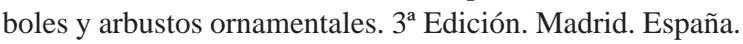
Mundi-Prensa. 53 p.

Caballer V. 1999. Valoración de árboles. Madrid, España. MundiPrensa. 247 p.

Chueca J. 2001. La Norma de Granada; un método de valoración económica de los árboles ornamentales. Consultado 25 oct. 2007. Disponible en http://www.drac.com/pers/chueca/ Granada.htm

Conover W. 1999. Practical nonparametric statistics. 3th Edition. New York, USA. John Wiley. 584 p.

Contato-Carol ML, E Ayuga-Tellez, MA Grande-Ortiz. 2008. A comparative analysis of methods for the valuation of urban trees in Santiago del Estero, Argentina. Spanish Journal of Agricultural Research 6(3): 314-352.

CTLA (Council of Tree and Landscape Appraiser, US). 2000. Guide for plant appraisal. $9^{\text {th }}$ Edition. International Society of Arboriculture, Champaign, IL. 143 p.

Cullen S. 2002. Tree appraisal: can depreciation factors be rated greater than 100\%? Journal of Arboriculture 28(3):153-158.

Cullen S. 2005. Tree appraisal: chronology of North American industry guidance. Journal of Arboriculture 31(4):157-162.

Cullen S. 2007. Putting a value on trees - CTLA guidelines and methods. Arboricultural Journal 30(1): 21 - 43.

Kirk R.1995. Experimental design: procedures for the behavioral sciences. Pacific Grove. Brooks/Cole. An International Thompson. California, USA. 921 p.

Konijnendijk C, S Syaka, T Randrup, L Schipperijn. 2004. Urban and peri-urban forestry in the development context: strategic and implementation. Journal of Arboriculture 30(5): 269-276.

Konijnendijk C, N Kjell, T Randrup, J Schipperijn. 2005. Urban forest and trees. Amsterdam, Holanda. Springer Verlag. $520 \mathrm{p}$.

Leal L, D Biondi, R Rochadelli. 2008. Investment on urban trees in the city of Curitiba: an approach based on the land income theory. Scientia Forestalis 36(78):141-149.

McPherson EG. 2007. Benefit-based tree valuation. Arboriculture 
\& Urban Forestry 33(1):1-11.

Ministerio del Interior (CL). 2004. Subsecretaria de Desarrollo Regional y Administrativo. Ley $N^{\circ} 18.695$ Orgánica Constitucional de Municipalidades. Consultado 18 oct. 2007. Disponible en http://www.subdere.gov.cl/1510/articles-65813_recurso_1.doc.

Moore GM. 1991. Amenity evaluation: A revised method. In The Scientific Management of Plants in the Urban Environment. Proceedings of the Burnley Centenary Conference. Centre of Urban Arboriculture, Melbourne, Australia. p. 166-171.

Municipalidad de Talca (CL). 2006. Informe de costos de mantenimiento de especies arbóreas urbanas. Talca. Departamento de aseo y ornato. Talca, Chile. Municipalidad de Talca. 12 p.

Nowak D, D Crane, J Dwyer. 2002. Compensatory value of urban trees in the United States. Journal of Arboriculture 28(4): 194-199.

Piolet S, N Jones, G García-Marín, JL García-Valdecantos, L Rivière, L Vidal Beaudet, M Bodson, T Raundrup. 2002. Tree establishment practice in towns and cities: results from
European survey. Urban Forestry and Urban Greening 1(2): 83-96.

Ponce-Donoso M, L Moya, O Bustos-Letelier. 2009. Evaluation of formula for the appraisal of urban trees in municipalities of Chile. Scientia Forestalis 37(83): 321-329.

Randrup TB. 2005. Development of Danish model for plant appraisal. Journal of Arboriculture 31(3):114-123.

Tyrväinen L. 2001. Economic valuation of urban forest benefits in Finland. Journal of Environmental Management 62:75-92.

Tyrväinen L, H Silvennoinen, O Kolehmainen. 2003. Ecological and aesthetic value in urban forest management. Urban Forestry and Urban Greening 1(3):135-150.

Watson G. 2001. A study of CTLA formula values. Journal o Arboriculture 27(6): 289-297.

Watson G. 2002. Comparing formula methods of tree appraisal. Journal of Arboriculture 28(1): 11-18.

Wenger K. 1984. Urban Forestry. In Wenger K ed. Forestry Handbook. Washington, United States. Wiley Interscience. p. 887-983. 
Anexo 1. Características de especies y fórmulas usadas en el estudio.

Characteristics of species and formulas used in the study.

Anexo 1A. Características de los árboles analizados. Selected species characteristics.

\begin{tabular}{|c|c|c|c|c|c|c|}
\hline $\mathrm{N}^{\mathrm{o}}$ & Árbol & $\begin{array}{c}\text { Edad } \\
\text { (años) }\end{array}$ & $\begin{array}{l}\text { DAP } \\
(\mathrm{cm})\end{array}$ & Ubicación** & Condición sanitaria & $\begin{array}{c}\text { Daño } \\
(\%)\end{array}$ \\
\hline 1 & Acer negundo L. & 25 & 44,6 & C. principal & Ataque patógeno & 70 \\
\hline 2 & Ailanthus altísima (Mill.) & 28 & 57,5 & Avda. principal & Buena & 60 \\
\hline 3 & Catalpa bignoniodes Walter & 25 & 22,0 & Avda. principal & Regular, pudrición & 25 \\
\hline 4 & Crytocarya alba (Mol.) Losser * & 105 & 57,3 & Plaza principal & Buena & 5 \\
\hline 5 & Ginkgo biloba L. & 105 & 87,9 & Plaza principal & Buena & 5 \\
\hline 6 & Grevillea robusta A. Cunn. ex R.Br & 18 & 35,9 & Avda. principal & Buena & 5 \\
\hline 7 & Jacaranda mimosifolia D. Don & 105 & 52,0 & Plaza principal & Buena & 10 \\
\hline 8 & Jubaea chilensis (Mol.) Baill * & 25 & 67,0 & Calle principal & Buena & 5 \\
\hline 9 & Liquidambar estyraciflua L. & 16 & 19,6 & Avda. principal & Buena & 20 \\
\hline 10 & Melia azedarach L. & 25 & 47,0 & Avda. principal & Buena & 8 \\
\hline 11 & Quercus robur L. & 5 & 4,5 & Avda. Secundaria & Regular & 5 \\
\hline 12 & Quillaja saponaria (Mol.) * & 25 & 57,3 & C. principal & Buena & 5 \\
\hline 13 & Schinus areira L. & 25 & 38,0 & C. secundaria & Buena & 5 \\
\hline 14 & Tilia americana L. & 5 & 4,8 & C. principal & Regular & 60 \\
\hline
\end{tabular}

* Especie nativa

** Avda. principal: calle de doble calzada, con alto flujo vehicular. C. principal: calle de una calzada con alto flujo vehicular. C. secundaria: calle de una calzada con bajo flujo vehicular. Plaza principal: plaza en el centro de la ciudad, donde se ubican los principales edificios públicos.

Anexo 1B. Precio y altura promedio al por menor de las especies en viveros locales.

Price and average height of species in retail local nurseries.

\begin{tabular}{ccc}
\hline Árbol $^{\circ}$ & Altura promedio $(\mathrm{m})$ & Precio minorista promedio (US\$) \\
\hline 1 & 2,5 & 3,4 \\
2 & 1,0 & 4,8 \\
3 & 1,5 & 3,1 \\
4 & 1,3 & 6,0 \\
5 & 1,8 & 20,8 \\
6 & 1,3 & 5,6 \\
7 & 1,5 & 10,8 \\
8 & 0,8 & 40,8 \\
9 & 1,8 & 12,1 \\
10 & 1,2 & 4,2 \\
11 & 1,3 & 5,7 \\
12 & 1,7 & 5,0 \\
13 & 0,9 & 2,9 \\
14 & 2,6 & 4,2 \\
\hline
\end{tabular}


Anexo 1C. Valor del árbol (VA) por grupos y rangos de edad.

Tree value (VA) by groups and age rank.

\begin{tabular}{|c|c|c|c|c|c|c|}
\hline \multirow[t]{2}{*}{ Edad (años) } & \multicolumn{3}{|c|}{ Valor en Municipalidad de Ñuñoa } & \multicolumn{3}{|c|}{ Valor en Municipalidad de Peñalolén } \\
\hline & Grupo 1 & Grupo 2 & Grupo 3 & Grupo 1 & Grupo 2 & Grupo 3 \\
\hline 1 a 4 & 1,00 & 1,00 & 1,00 & 0,08 & 0,17 & 0,33 \\
\hline 5 a 10 & 1,50 & 2.00 & 2.80 & 0,33 & 0,50 & 0,99 \\
\hline 11 a 15 & 2,40 & 3,51 & 4,91 & 1,32 & 1,49 & 2,97 \\
\hline 16 a 20 & 4,08 & 6,16 & 8,62 & 3,96 & 4,46 & 8,91 \\
\hline 21 a 25 & 7,34 & 10,81 & 15,14 & 11,89 & 13,37 & 26,74 \\
\hline 26 a 30 & 11,75 & 16,22 & 22,70 & 14,26 & 16,05 & 32,09 \\
\hline 31 a 35 & 18,21 & 24,32 & 34,05 & 9,98 & 19,25 & 38,51 \\
\hline 36 a 40 & 25,50 & 34,05 & 47,68 & 6,90 & 23,10 & 46,21 \\
\hline 41 a 45 & 33,15 & 45,97 & 64,36 & 4,98 & 27,73 & 55,45 \\
\hline 46 a 50 & 39,78 & 59,77 & 83,67 & 3,42 & 33,27 & 65,54 \\
\hline 51 a 55 & 45,74 & 71,72 & 100,41 & 2,40 & 39,93 & 69,87 \\
\hline 56 a 60 & 50,32 & 82,48 & 120,49 & 1,68 & 47,90 & 73,36 \\
\hline 61 a 65 & 55,35 & 90,72 & 138,56 & 1,18 & 33,54 & 77,03 \\
\hline 66 a 70 & 60,89 & 99,80 & 152,42 & 1,18 & 23,48 & 80,88 \\
\hline 71 a 75 & 65,76 & 107,78 & 166,13 & 1,18 & 16,43 & 84,93 \\
\hline 76 a 80 & 70,42 & 114,25 & 177,76 & 1,18 & 11,50 & 89,17 \\
\hline 81 a 85 & 74,65 & 119,96 & 186,65 & 1,18 & 9,05 & 93,63 \\
\hline 86 a 90 & 78,38 & 123,56 & 192,25 & 1,18 & 5,64 & 98,31 \\
\hline 91 a 95 & 79,95 & 126,03 & 196,10 & 1,18 & 3,95 & 103,23 \\
\hline 96 a 100 & 80,75 & 127,29 & 198,06 & 1,18 & 2,76 & 108,39 \\
\hline
\end{tabular}


Anexo 1D. Valores de cada variable por fórmula.

Variables' values of each formula.

\begin{tabular}{rrrrrrrrrrrrrrrrr}
\hline Árbol & \multicolumn{1}{c}{ COPIMA } & \multicolumn{1}{c}{ Ñunoa } & \multicolumn{1}{c}{ Peñalolén } \\
\cline { 2 - 3 } & C1 & C2 & C3 & C4 & $\tilde{N} 1$ & $\tilde{N} 2$ & $\tilde{N} 3$ & P1 & P2 & P3 & CT1 & CT2 & CT3 & CT4 & CT5 \\
\hline 1 & 6,87 & 3 & 10 & 12 & 500,88 & 0,60 & 0,80 & 1 & 0,80 & 811,37 & 242,15 & $1.622,59$ & 0,85 & 0,2 & 0,6 \\
2 & 7,00 & 10 & 8 & 12 & 801,82 & 0,10 & 0,50 & 1 & 0,80 & 973,10 & 402,49 & $2.817,38$ & 0,50 & 0,8 & 0,6 \\
3 & 4,67 & 4 & 8 & 6 & 500,88 & 0,30 & 0,01 & 1 & 0,80 & 912,37 & 58,92 & 263,13 & 0,45 & 0,8 & 0,6 \\
4 & 8,67 & 6 & 10 & 12 & $13.650,73$ & 0,02 & 0,05 & 2 & 0,04 & 188,34 & 399,70 & $3.463,70$ & 0.85 & 0,6 & 1,0 \\
5 & 30,00 & 8 & 10 & 18 & $13.650,73$ & 0,10 & 0,10 & 2 & 0,05 & 188,34 & 940,59 & $28.217,00$ & 0,75 & 1,0 & 0,8 \\
6 & 9,47 & 8 & 8 & 9 & 278,42 & 0,05 & 0,05 & 1 & 0,05 & 304,35 & 156,90 & $1.485,14$ & 0,60 & 0,8 & 0,6 \\
7 & 15,60 & 9 & 10 & 12 & $8.772,93$ & 0,10 & 0,10 & 2 & 0,10 & 188,34 & 329,18 & $5.135,03$ & 0,40 & 0,8 & 0,8 \\
8 & 58,67 & 9 & 10 & 15 & $1.033,15$ & 0,00 & 0,40 & 1 & 0,05 & $1.824,74$ & 546,48 & $32.058,85$ & 0,90 & 1,0 & 0,6 \\
9 & 17,33 & 8 & 8 & 6 & 420,36 & 0,10 & 0,15 & 1 & 0,80 & 304,35 & 46,77 & 810,63 & 0,70 & 0,8 & 0,6 \\
10 & 6,00 & 8 & 8 & 12 & 500,88 & 0,05 & 0,10 & 1 & 0,80 & 811,37 & 268,92 & $1.613,46$ & 0,60 & 0,8 & 0,6 \\
11 & 8,13 & 6 & 8 & 1 & 191,07 & 0,05 & 0,02 & 2 & 0,04 & 34,12 & 2,47 & 20,05 & 0,65 & 0,4 & 0,8 \\
12 & 7,13 & 9 & 8 & 12 & $1.033,15$ & 0,02 & 0,01 & 1 & 0,05 & 912,37 & 399,70 & $2.851,37$ & 0,80 & 1,0 & 0,6 \\
13 & 4,13 & 4 & 8 & 9 & $1.033,15$ & 0,40 & 0,60 & 1 & 0,05 & 912,37 & 175,79 & 726,69 & 0,55 & 0,4 & 0,8 \\
14 & 6,00 & 9 & 8 & 1 & 191,07 & 0,30 & 0,60 & 1 & 0,80 & 22,52 & 5,14 & 16,83 & 0,70 & 0,8 & 0,6 \\
\hline
\end{tabular}

\begin{tabular}{|c|c|c|c|c|c|c|c|c|c|c|c|c|c|c|c|}
\hline \multirow{2}{*}{ Árbol } & \multicolumn{5}{|c|}{ Burnley } & \multicolumn{7}{|c|}{ Helliwell** } & \multicolumn{3}{|c|}{ STEM ${ }^{* * *}$} \\
\hline & B1 & B2 & B3 & B4 & B5 & H1 & $\mathrm{H} 2$ & H3 & $\mathrm{H} 4$ & H5 & H6 & H7 & S1 & S2 & S3 \\
\hline 1 & 16,62 & 1,03 & 0,6 & 0,1 & 0,8 & 2,0 & 2 & 0,5 & 3 & 2 & 0,5 & 1 & 74 & 6,87 & 0 \\
\hline 2 & 883,55 & 1,05 & 0,7 & 0,7 & 0,9 & 4,0 & 2 & 1,0 & 3 & 1 & 2,0 & 1 & 122 & 7,00 & 0 \\
\hline 3 & 10,18 & 0,69 & 0,8 & 0,7 & 0,8 & 1,5 & 2 & 1,0 & 2 & 2 & 3,0 & 1 & 92 & 4,67 & 0 \\
\hline 4 & $1.194,56$ & 1,30 & 1,0 & 0,9 & 0,9 & 4,0 & 3 & 3,0 & 1 & 3 & 4,0 & 3 & 178 & 8,67 & 4 \\
\hline 5 & 445,31 & 4,50 & 1,0 & 0,9 & 0,9 & 4,0 & 4 & 2,0 & 1 & 2 & 3,0 & 2 & 178 & 30,00 & 4 \\
\hline 6 & 663,65 & 1,42 & 0,7 & 0,8 & 0,9 & 2,5 & 2 & 1,0 & 3 & 1 & 3,0 & 1 & 107 & 9,47 & 0 \\
\hline 7 & 475,15 & 2,34 & 0,8 & 0,6 & 0,9 & 4,0 & 2 & 2,0 & 1 & 3 & 3,0 & 2 & 182 & 15,60 & 2 \\
\hline 8 & 318,08 & 8,80 & 0,9 & 0,7 & 0,9 & 2,5 & 3 & 3,0 & 3 & 3 & 4,0 & 2 & 146 & 58,67 & 0 \\
\hline 9 & 7,07 & 2,60 & 0,8 & 0,8 & 0,8 & 1,0 & 2 & 1,0 & 3 & 1 & 2,0 & 1 & 94 & 17,33 & 0 \\
\hline 10 & 129,02 & 0,90 & 0,8 & 0,8 & 0,8 & 2,5 & 2 & 2,0 & 2 & 3 & 2,0 & 1 & 104 & 6,00 & 0 \\
\hline 11 & 0,75 & 1,22 & 0,6 & 0,3 & 0,9 & 0,5 & 2 & 0,5 & 3 & 1 & 0,5 & 1 & 58 & 8,13 & 0 \\
\hline 12 & 115,45 & 1,07 & 1,0 & 0,8 & 0,9 & 2,5 & 2 & 2,0 & 1 & 2 & 3,0 & 1 & 134 & 7,13 & 0 \\
\hline 13 & 339,28 & 0,62 & 0,8 & 0,6 & 0,5 & 2,5 & 2 & 1,0 & 2 & 1 & 0,5 & 1 & 98 & 4,13 & 0 \\
\hline 14 & 22,90 & 0,90 & 1,0 & 0,8 & 0,9 & 1,0 & 3 & 2,0 & 2 & 3 & 3,0 & 1 & 95 & 6,00 & 0 \\
\hline
\end{tabular}

* En dólares americanos 1 US $\$=500$ pesos chilenos.

** El monto en dinero correspondió a un valor único de US\$ 21,20.

*** Se usó como factor de conversión 2; el costo de plantación es US\$ 0,996 y el costo de mantención es de US\$1,44.

C1: precio minorista, C2: valor estético y sanidad, C3: índice de situación, C4: índice de dimensión, Ñ1: valor actual, Ñ2: daño causado, Ñ3: daño presente, P1: factor de ubicación, P2: estado del árbol, P3: valor del árbol, CT1: área del tronco, CT2: precio base, CT3: especie, CT4: condición, CT5: localización, B1: volumen del árbol, B2: valor base, B3: expectativa de vida, B4: forma y vigor, B5: localización, H1: tamaño, H2: expectativa de vida, H3: importancia en el espacio, H4: presencia de otros árboles, H5: relación con el entorno, H6: forma, H7: factores especiales, S1: sumatoria de puntos, S2: costo mayorista y S3: puntos de notabilidad. 
Anexo 2. Grupos definidos en la ordenanza de Ñuñoa.

Groups defined in the ordinance of Ñuñoa.

o Grupo 1: Acacia dealbata Link, Acacia longifolia Andrews Willd, Acacia melanoxylon R. Br., Acacia retinodes Schltdl., Acer negundo L., Ailanthus altisima Mill., Albizzia lophanta Willd., Betula pendula Roth., Catalpa bignonioides Walter, Ceratonia siliqua L., Crataegus oxyacantha L., Eucalyptus spp., Gleditsia triacanthos L., Grevillea robusta A. Cunn., Ligustrum lucidum Ait., Melia azedarach L., Morus alba L., Morus nigra L., Myoporum tenuifolium G. Forst., Parkinsonia aculeata L., Paulownia tormentosa Stend, Pinus radiata D. Don, Populus spp., Prunus spp., Styphnolobium japonicum (L.) Schott. y Tamarix gallica L.

o Grupo 2: Acer palmatum Thunb., Acer pseudoplatanus L., Bauhinia candicans Benth., Brachychiton acerifolium A. Cunn. ex F.L. Muell., Brachychiton populneum Shott et Endl., Casuarina equisetifolia L., Cersis siliquastrum L., Eleagnus angustifolia L., Erythrina umbrosa H.B. et K., Fraxinus americana L., Fraxinus excelsior L., Fraxinus ornus L., Jacaranda mimosifolia D. Don, Koelreuteria paniculada Laxm., Lagerstromeria indica (L.) Pers., Laurus nobilis L., Liquidambar styraciflua L., Robinia pseudoacacia L., Sophora microphylIa L., Ulmus americana Planch. y Ulmus procera Salisb.

o Grupo 3: Abies spp., Acacia caven (Mol.) Mol., Aesculus hippocastanum L., Araucaria angustifolia (Bertol.) Kuntze, Araucaria araucana (Molina) K. Koch, Araucaria bidwillii Hook f., Araucaria columnaris (G. Forst) Hook f., Araucaria excelsa (Lamb.), Azara spp., Beilschmiedia miersii (Gay) Kosterm., Castanea sativa Mill., Cedrus atlantica (Endl.) Carrière, Cedrus deodara (D. Don) G. Don, Cedrus libani A. Rich., Celtis australis L., Chamaecyparis lawsoniana (A. Murray) Parl., Cinamomum camphora L., Crinodendron patagua Mol., Cryptocarya alba (Mol.) Looser, Cryptomeria japonica (L. f.) D. Don, Cupressus macrocarpa Hartw., Cupressus sempervirens L., Drimys winteri J.R. et G. Forster, Fagus sylvatica L., Fagus sylvatica var. Ferruginea (Aiton) N. Coleman, Ginkgo biloba L., Jubaea chilensis (Mol.) Baillon, Juglans nigra L., Juglans regia L., Liriodendron tulipifera L., Magnolia grandiflora L., Maytenus boaria Mol., Persea lingue (R. et P.) Nees ex Koop, Phoenix reclinata Jacq., Phytolacca dioica L., Platanus occidentalis L., Platanus orientalis L., Platanus acerifolia (Dryand.) Willd., Prosopis chilensis (Mol.) Stuntz, Quercus spp., Quillaja saponaria Mol., Salix humboldtiana Willd., Schinus areira L., Schinus latifolius (Gill. ex Lindl.) Engel, Schinus molle L., Sequoia sempervirens (D. Don) Endl., Taxodium distichum (L.) L. Rich., Taxus baccata L., Thuja orientalis (Endl.), Tilia spp., Trachycarpus fortunei (Hook.) H. Wendl., Washingtonia filifera (Lindl.) H. Wendl. y Washingtonia robusta $\mathrm{H}$. Wendl.

Anexo 3. Grupos definidos en la ordenanza de Peñalolén. Groups defined in the ordinance of Peñalolén.

o Grupo 1: Acacia caven (Mol.) Mol, Acacia dealbata Link, Acacia melanoxylon R. Br., Acacia retinodes Schltdl., Acer negundo L., Ailanthus altisima Mill., Albizzia lophanta Hill., Betula pendula Roth., Catalpa bignonioides Walter, Ceratonia siliqua L., Crataegus oxyacantha L., Eucalyptus spp., Gleditsia triacanthos L., Grevillea robusta A. Cunn. ex R.Br., Ligustrum lucidum Ait., Melia azedarach L., Myoporum tenuifolium G. Forst., Parkinsonia aculeata L., Paulownia tormentosa Stend, Pinus radiata D. Don, Platanus orientalis L., Phytolacca dioica L., Populus spp., Prunus spp., Sophora microphylIa Ait., Styphnolobium japonicum (L.) Schott, Tamarix gallica L., Tilia spp, Salix spp y Ulmus procera Salisb.

o Grupo 2: Acer sempervirens L., Aesculus hippocastanum L., Acer pseudoplatanus L., Bauhinia candicans Benth., Brachychiton acerifolium A. Cunn. ex F.L. Muell, Brachychiton populneum Shott et Ende., Casuarina equisetifolia L., Cersis siliquastrum L., Crinodendron patagua Mol., Cyptocaria alba (Mol.) Looser, Eleagnus angustifolia L., Erythrina umbrosa H.B. et K., Fraxinus americana L., Fraxinus excelsior L., Fraxinus ornus L., Ginkgo biloba L., Jacaranda mimosifolia D. Don, Koelreuteria paniculada Laxm., Lagerstromeria indica (L.) Pers., Laurus nobilis L., Liquidambar styraciflua L., Peumus boldus Mol., Quillaja saponaria Mol., Quercus robur L., Robinia pseudoacacia L., Schinus areira L., Sophora microphylIa L., Ulmus americana Planch. y Ulmus procera Salisb.

o Grupo 3: Araucaria araucana (Molina) K. Koch, Fagus sylvatica L., Jubaea chilensis (Mol.) Baillon, Magnolia grandiflora L. y Sequoia sempervirens (D. Don) Endl. 
